\title{
The chemical composition of the mild barium star HD 202109^
}

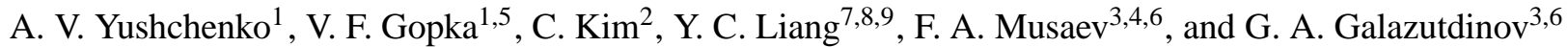 \\ 1 Odessa Astronomical observatory, Odessa National University, Park Shevchenko, Odessa 65014, Ukraine \\ e-mail: yua@odessa.net,gopka@arctur.tenet.odessa.ua \\ 2 Department of Earth Science Education, Chonbuk National University, Chonju 561-756, Korea \\ e-mail: chkim@astro.chonbuk.ac.kr \\ 3 The International Centre for Astronomical, Medical and Ecological Research of the Russian Academy of Sciences and the \\ National Academy of Sciences of Ukraine, Golosiiv, Kiev 03680, Ukraine (ICAMER) \\ 5 Isaac Newton Institute, Santiago, Chile, Odessa branch, Ukraine \\ ${ }^{6}$ Isaac Newton Institute, Santiago, Chile, SAO branch, Russia \\ 7 National Astronomical Observatories, Chinese Academy of Sciences, 100012 Beijing, PR China \\ ${ }^{8}$ GEPI, Observatoire de Paris-Meudon, 92195 Meudon, France \\ 9 Institut für Astronomie und Astrophysik der Universität München, Universitäts-Sternwarte München, Scheinerstr. 1, \\ 81679 München, Germany
}

Received 27 February 2003 / Accepted 7 August 2003

\begin{abstract}
We present chemical abundances of the mild barium star HD 202109 ( $\zeta$ Cyg) determined from the analysis of a spectrum obtained by using the 2-m telescope at the Peak Terskol Observatory and a high-resolution spectrometer with $R=80000$, signal to noise ratio $>100$. We also present the atmospheric parameters of the star determined using various methods including iron-line abundance analysis. For line identifications, we use whole-range synthetic spectra computed from Kurucz's database and the latest lists of spectral lines. Among the determined abundances of 51 elements, those of P, S, K, $\mathrm{Cu}, \mathrm{Zn}, \mathrm{Ge}$, $\mathrm{Rb}, \mathrm{Sr}, \mathrm{Nb}, \mathrm{Mo}, \mathrm{Ru}, \mathrm{Rh}, \mathrm{Pd}, \mathrm{In}, \mathrm{Sm}, \mathrm{Gd}, \mathrm{Tb}, \mathrm{Dy}, \mathrm{Er}, \mathrm{Tm}, \mathrm{Hf}, \mathrm{Os}, \mathrm{Ir}, \mathrm{Pt}, \mathrm{Tl}$, and Pb were not investigated previously. Assuming that the overabundance pattern of Ba stars is due to binary accretion, the observed abundance pattern of the neutron-capture process elements in HD 202109 can be explained by combining the AGB star nucleosynthesis and the wind accretion scenario.
\end{abstract}

Key words. line: identification - stars: abundances - stars: atmospheres - stars: evolution - stars: binaries stars: individual: HD 202109

\section{Introduction}

Barium stars were first defined by Bidelman \& Keenan (1951). Burbidge \& Burbidge (1957) obtained the first quantitative result about the chemical composition of a barium star HD 46407 and reported overabundance of the heavy elements. Burbidge et al. (1957) interpreted the overabundance in terms of the $s$-process. Another important finding in understanding the physical process of the formation of barium stars was the fact that these stars are members of binary systems (McClure et al. 1980). Wallerstein et al. (1997) revieved the development of the theory of element creation in stars.

Send offprint requests to: A. V. Yushchenko,

e-mail: yua@mail.chonbuk.ac.kr

* Based on observations obtained at the 2-m telescope of Peak Terskol observatory near Mt. Elbrus, Northern Caucasus, Russia - International Center for Astronomical, Medical and Ecological Research (ICAMER), Ukraine \& Russia.
To explain the process of stellar evolution from abundance analysis, it is essential to construct detailed observed abundance patterns based on abundances of a large number of elements. Unfortunately, detailed abundance patterns are known only for few stars including the Sun, for which abundances of 73 chemical elements are known (Grevesse \& Sauval 1998). Some other well-determined stellar abundance patterns are those for GS 22892-05 (based on 57 elements; Sneden et al. 2003), GS 31082-001 (based on 38 elements with $Z>38$; Aoki et al. 2003; Sneden et al. 2000), Procyon (based on 55 elements; Yushchenko \& Gopka 1996a,b), Przybylski's star (based on 54 elements; Cowley et al. 2000), and $\chi$ Lupi (based on 51 elements; Leckrone et al. 1999).

Theoretical abundance patterns of $s$-process elements in barium stars were published, for example, by Cowley \& Downs (1980), Malaney (1987), Liang et al. (2000). The hypothesis that the overabundances of heavy elements in the atmospheres of barium stars are produced by accreting the ejecta from their 
AGB companion progenitor seems to be the most reliable explanation. These AGB companions have quickly evolved into white dwarfs and cannot be easily detected.

In this paper, we attempt to construct the detailed abundance pattern of one of the barium stars by determining abundances of as many elements as possible, paying special attention to $s$-process elements. We used high resolution observations in the blue spectral region, the latest atomic data, and the most updated spectrum synthesis method for better line identifications.

The star selected for our investigation is HD 202109 ( $\zeta$ Cyg, BS 8115, 64 Cyg), which is a prototype mild barium star. The star was first noticed as a barium star by Bidelman. The first quantitative abundance analysis for this star was done by Chromey et al. (1969). Keenan \& MacNeil (1976) determined the star's spectral class as G8+III-IIIa Ba 0.6. Griffin \& Keenan (1992) reclassified the spectral class as G8+IIIa Ba 0.6 and published the orbit of the bright component of this spectroscopic binary. Griffin (1996) pointed out that the orbital period of the system ( $\sim 18$ years) is the longest among the systems with moderate enhancement of $s$-process elements. Pourbaix $\&$ Jorissen (2000) investigated the HIPPARCOS transit data of HD 202109, but its long period did not permit them to find a reliable orbit. The white dwarf companion was directly imaged in recent HST observations by Barstow et al. (2001).

Previous studies on the chemical composition of the star include those of Sneden et al. (1981), Gratton (1985), Berdyugina (1993) (CNO elements), Zacs (1994), and Boyarchuk et al. (2001) (heavier elements). Cohen et al. (1999) and Garetta et al. (2001) used HD 202109 as a comparison star and published the abundances of several elements.

We select HD 202109 as our target for the following reasons. First, since chemical abundances are known for some elements from previous studies, a comparison with the previous results allows us to derive more reliable abundance pattern. Second, the star is bright enough for us to acquire highquality spectra using a mid-size telescope. Third, HD 202109 is an ideal target to test the accretion hypothesis. Can we apply this hypothesis to barium stars with moderate enhancement of $s$-process elements, which is expected for barium stars with slow orbital motions? Fourth, since the star was observed in a wide range of wavelengths from $\mathrm{X}$-ray to radio, we can derive a more concrete model of this binary system.

\section{Observations and data reduction}

A high resolution spectrum of HD 202109 was obtained on September 19 in 2000 using a coude-echelle spectrometer (Musaev et al. 1999) mounted on the 2-m "Zeiss" telescope at the Peak Terskol Observatory located near Mt. Elbrus (Northern Caucasus, Russia) 3124 m above sea level.

We used one of the medium-resolution modes of the spectrograph with a resolving power of $R=80000$. The observed wavelength range, $\lambda \lambda 3495-10000 \AA \AA$, was covered by 85 echelle orders. In the observed spectrum, there are gaps between the orders in the wavelength region of $\lambda \geq 3780 \AA$ and the width of each gap increases from $0.5 \AA$ to $125 \AA$ as the wavelength increases. The CCD, made by Wright Instruments, has a $1242 \times 1152$ format and each pixel has a physical size of $22 \mu \mathrm{m}$. The signal-to-noise ratio of the spectrum is not less than 100 over the whole wavelength range and reaches 300 or more in the blue and red parts of the spectrum.

The first-stage data processing (background subtraction, echelle vector extraction from the echelle-images, and wavelength calibration) was done using the latest version of PCbased DECH software (Galazutdinov 1992). For other processes including continuum placement, we use URAN software developed by one of us (Yushchenko 1998). The location of the continuum was determined taking into account the calculated spectrum. More details about this procedure were discussed for example by Walgren (1995).

\section{Atmospheric parameters}

With the processed data, we then determined the atmospheric parameters including the effective temperature, $T_{\text {eff }}$, the surface gravity, $\log g$, and the microturbulence, $v_{\text {micro }}$.

\subsection{Effective temperature from colors}

We first determined $T_{\text {eff }}$ of HD 202109 from color information. For this, we use two colors of $V-K$ and $b-y$. Following the calibrations of Di Benedetto (1998) for $V-K$ and Alonco et al. (1999) for $b-y$, we find that the temperatures determined from these two colors are $T_{\text {eff }}=4927 \mathrm{~K}$ and $4903 \mathrm{~K}$, respectively.

We compare our results of $T_{\text {eff }}$ with previous determinations based on different methods and data. Andrievsky et al. (2002) determined $T_{\text {eff }}$ of the star by using different photometric data and calibrations. They found five values of $T_{\text {eff }}=$ $5130 \mathrm{~K}, 5070 \mathrm{~K}, 4900 \mathrm{~K}, 5120 \mathrm{~K}$, and $5100 \mathrm{~K}$ and presented $T_{\text {eff }}=5100 \mathrm{~K}$ as a representative value. Gray \& Brown (2001) used the line-ratio method, and found the value $T_{\text {eff }}=4987 \mathrm{~K}$. Gray \& Brown (2001) used HD 202109 as one of the calibrating stars to set their temperature scale. Upon our request, V. Kovtyukh kindly provided his determination based on the depth ratios of the iron lines. Using the latest version of the Kovtyukh \& Gorlova (2000) method, he found $T_{\text {eff }}=5044 \pm$ $44 \mathrm{~K}$.

For the effective temperature one could use the temperature determined from color information, but we decided to choose the value determined from the iron-line abundance analysis because it is known that the precision of $T_{\text {eff }}$ determined from spectroscopic determinations is significantly higher than that of the temperature determined from color information (Boyarchuk 2001).

\subsection{Atmospheric parameters from iron line abundance analysis}

Usually atmospheric parameters from iron line analysis are determined by investigating the correlation(s) of the observed equivalent widths, $\log \left(W_{\lambda} / \lambda\right)$, (and the excitation potential, $\left.E_{\text {low }}\right)$ of iron lines with the iron abundances calculated based on the equivalent widths of the individual lines by assuming a certain atmosphere model. In this process, it is customary to use fixed values of $T_{\text {eff }}$ and $\log g$, under the assumption that these parameters can be constrained from other information 
(for example from colors), and leave $v_{\text {micro }}$ as the only free parameter. Variation of this parameter makes it possible to avoid the influence of the uncertainties in effective temperature and surface gravity.

Yushchenko et al. (1999) noticed that the scattering information of the iron abundances derived from different iron lines is useful in determining correct atmospheric parameters. The scattering for models with wrong parameters is usually larger than for models with right parameters.

In our analysis, we therefore determine the atmospheric parameters not only by leaving all of them as free parameters but also by using the additional information of scattering in the derived iron abundances.

We selected 89 clean Fe I lines in the synthetic spectrum of HD 202109 and measured their equivalent widths in the observed spectrum by fitting their profiles with a gaussian.

We used solar oscillator strengths (Gurtovenko \& Kostik 1989). For iron lines where oscillator strengths were not available in Gurtovenko \& Kostik (1989), we used solar values computed by the spectrum synthesis method. Details of the line selection and the synthetic spectrum calculations are described in Sect. 4. Once oscillator strengths are set, the iron abundances are derived from the individual lines by using the WIDTH9 code of Kurucz (1995).

HD 202109 is a binary system. A full description of binary systems requires the determination of two sets of atmospheric parameters for the individual components of the system and the flux ratio between them. We note, however, that although HD 202109 is a binary, the flux from the white dwarf can be neglected in our case. For determination of atmospheric parameters of a binary where the fluxes of the component stars are of the same order, see Yushchenko et al. (1999).

For atmosphere models Kurucz's (1995) data base was used. We made a $21 \times 6$ subgrid of atmosphere models by subdividing the grid of Kurucz's data base. The ranges (and intervals) of the individual parameters are $4750 \mathrm{~K} \leq T_{\text {eff }} \leq$ $5250 \mathrm{~K}\left(\Delta T_{\text {eff }}=25 \mathrm{~K}\right)$ for the effective temperature and $2.5 \leq \log g \leq 3.0(\Delta \log g=0.1)$ for the surface gravity. Calculations for all models were made with 41 values of the microturbulent velocity $1.0 \mathrm{~km} \mathrm{~s}^{-1} \leq v_{\text {micro }} \leq 3.0 \mathrm{~km} \mathrm{~s}^{-1}$ $\left(\Delta v_{\text {micro }}=0.05 \mathrm{~km} \mathrm{~s}^{-1}\right)$

Based on each set of atmosphere parameters, we compute iron abundances corresponding to the individual lines. For iron abundances computed for each set of atmosphere parameters, we then calculate the scattering of the abundances and the coefficient(s) of the correlation(s) between the equivalent widths (and the excitation potentials) and the iron abundances.

Among the tested models, the best atmosphere model was chosen to be the one providing zero (or very close to zero) correlation coefficients and minimal scattering in the derived iron abundances. We find that the best model has atmosphere parameters $T_{\text {eff }}=5050 \mathrm{~K}, \log g=2.8$, and $v_{\text {micro }}=1.45 \mathrm{~km} \mathrm{~s}^{-1}$. We note that our determination of $T_{\text {eff }}$ is very close to the value of Kovtyukh determined by using the line-depth ratio method. We present the correlation between $\log N(\mathrm{Fe})$ and $\log \left(W_{\lambda} / \lambda\right)$ in Fig. 1 and the correlation between $\log N(\mathrm{Fe})$ and $E_{\text {low }}$ in Fig. 2 for the best atmosphere model. In Table 1, we compare our final atmospheric parameters with those of other determinations.

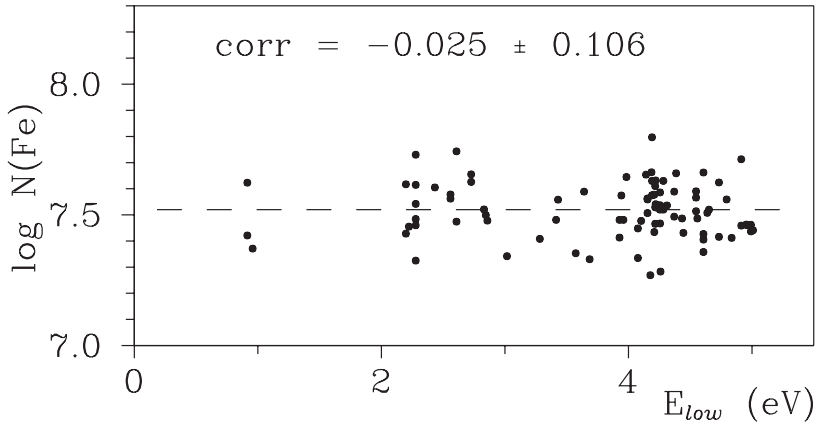

Fig. 1. The correlation between the iron abundances determined from $89 \mathrm{Fe}$ I lines in the spectrum of HD 202109 and the excitation energies, $E_{\text {low }}$, of the individual lines. Our final atmosphere parameters and abundances of other chemical elements were used.

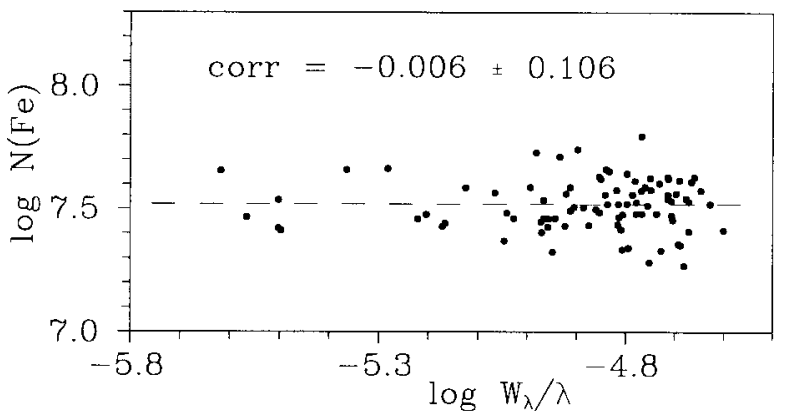

Fig. 2. The correlation between the iron abundances determined from $89 \mathrm{Fe} I$ lines in the spectrum of HD 202109 and the equivalent widths, $\log \left(W_{\lambda} / \lambda\right)$, of the individual lines.

Note that since our method uses scattering information of iron abundances, it enables us to determine the iron abundance. We found that the iron abundance of HD 202109 is $+0.01 \pm$ 0.11 dex relative to the solar value.

\section{Abundance analysis}

\subsection{Synthetic spectrum}

We calculated the abundances of all elements except iron by using the synthetic spectrum method. To construct accurate synthetic spectra one needs to find the parameters of line broadening.

The magnetic field of HD 202109 was investigated by Tarasova (2002). She found values of $-5.4 \pm 0.2,-0.4 \pm 2.0$ and $0.2 \pm 1.8$ Gauss. A field of this strength cannot influence our final final abundances significantly. The rotation velocity was determined by Gray (1989) $v_{\text {rot }}=3.4 \pm 0.5 \mathrm{~km} \mathrm{~s}^{-1}$ and Medeiros \& Mayor (1999) $v_{\text {rot }} \lesssim 1.0 \mathrm{~km} \mathrm{~s}^{-1}$.

We assume that lines are broadened mainly by the macroturbulence. We estimate the macroturbulent velocity by analyzing the profiles of iron lines with accurate oscillator strengths. We find that $v_{\text {macro }}=3.2-3.5 \mathrm{~km} \mathrm{~s}^{-1}$. This value includes all possible line broadening mechanisms. We used a Gaussian model of macroturbulence (Gray 1976).

With the input of all these parameters, the synthetic spectra are constructed using the SYNTHE code of Kurucz (1995). We used various lists of spectral lines. These include all atomic and 
Table 1. Atmospheric parameters of HD 202109 ( $\zeta$ Cyg).

\begin{tabular}{lcll}
\hline \hline \multicolumn{1}{c}{ reference } & $\begin{array}{c}T_{\text {eff }} \\
(\mathrm{K})\end{array}$ & $\log g$ & $\begin{array}{c}v_{\text {micro }} \\
\left(\mathrm{km} \mathrm{s}^{-1}\right)\end{array}$ \\
\hline Chromey (1969) & 5143 & & \\
Pilachowski (1977) & 4893 & 2.8 & \\
Sneden et al. (1981) & 4870 & 2.5 & 2.0 \\
& 5000 & 2.8 & 2.2 \\
Gratton et al. (1982) & 4941 & 2.0 & \\
Gratton (1985) & 4950 & 2.0 & 2.4 \\
Fernandez-Villacanas & 4900 & 2.0 & \\
et al. (1990) & & & \\
McWilliam (1990) & 4990 & 2.87 & \\
Berdyugina (1993) & 5000 & 2.7 & 2.0 \\
Berdyugina \& Savanov (1994) & 5000 & 2.8 & \\
Zacs (1994) & 5050 & 2.8 & 3.5 \\
Cohen et al. (1999) & 4950 & 2.7 & 1.6 \\
Boyarchuk et al. (2001) & 4977 & 2.52 & 1.4 \\
Gray \& Brown (2001) & 4987 & & \\
Andrievsky et al. (2002) & 5100 & 2.5 & 1.5 \\
This paper & 5050 & 2.8 & 1.45 \\
\hline
\end{tabular}

molecular lines of Kurucz's database (1995), Morton's (2000) lines, the DREAM database (Biemont et al. 2002) lines for lanthanides and actinides, part of the lines from VALD database (Piskunov et al. 1995), and lines in other lists. We took into account the hyperfine-structure lines and isotopic splitting of the lines for $\mathrm{Mn}, \mathrm{Cu}$, and $\mathrm{Eu}$. Split-line data are taken from the Kurucz database (1995).

\subsection{Line identifications}

To better identify heavy element lines, the synthetic spectrum used for line identifications is produced by increasing the abundances of uninvestigated heavy elements by an amount of +0.5 dex compared to the solar system values.

For identification of lines of any element we selected all not-strongly-blended lines of this element in the results of synthetic spectrum calculations and inspected these lines in the observed spectrum to select the best lines for abundance analysis. The observed and synthetic spectra of HD 202109 and of the Sun were displayed simultaneously on the computer screen. This permits to avoid identification errors and to increase the number of selected lines. The URAN code of Yushchenko (1998) was used.

In Figs. 3 and 4, we present a part of the observed spectrum of HD 202109 and its approximation by the synthetic one.

\subsection{Abundance determinations}

Once the individual lines are identified, we determine the element abundances. For line identifications and abundance determinations we used the URAN code of Yushchenko (1998). In this code abundances are computed in semiautomatic mode. To approximate the observed spectrum by a synthetic one we changed the oscillator strengths of all lines in the investigated

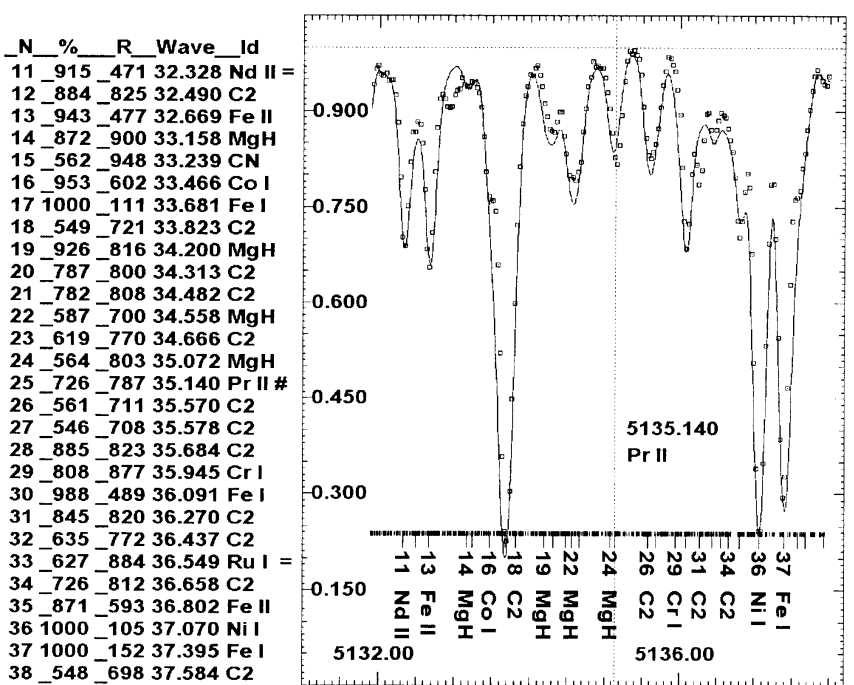

Fig. 3. Part of the spectrum of HD 202109. The squares are the observed spectrum. The solid line is the synthetic one. The positions of the strong and faint lines in the synthetic spectrum are shown by long and short dashes at the bottom of the figure. Part of the strong lines are marked by numbers and identification. A table with line data is in the left of the figure. The first column is the line number. The second column is the portion of the line in the total line absorption coefficient at the wavelength of the center of the line in the synthetic spectrum. For a clean line the value in this column must be 1000. The third column is the value of synthetic spectrum at the center of the line. The continuum value is 1000 . Only strong lines are listed. The values of the synthetic spectrum in the table are not smoothed by instrumental and macroturbulence profiles. In the last two columns we give the last digits of the wavelengths and the identifications. Lines of $r-, s$ process elements are marked by an equal sign. The Pr II $\lambda 5135.140$ line is marked by a \# sign in the table and by a vertical dashed line in the spectrum. This and the next figures are the PrintScreen output of URAN software (Yushchenko 1998).

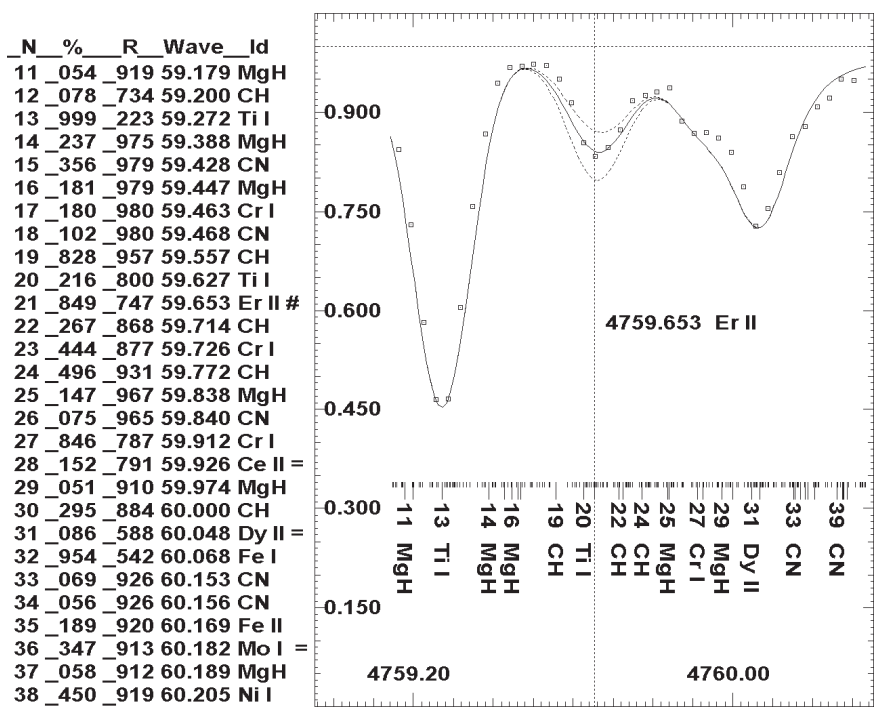

Fig. 4. Observed (squares) and calculated spectra of HD 202109. The solid line is the spectrum, calculated with our final abundances. Dashed lines are the spectra, calculated with erbium abundances changed by \pm 0.2 dex from the best value. The central line of the plot (Er II $\lambda 4759.653$ ) is marked by a \# sign in the table and by a vertical dashed line in the spectrum. 
Table 2. Fe I and Fe II lines in the spectrum of HD 202109 (example, full table is available in electronic form).

\begin{tabular}{ccrccc}
\hline \hline $\begin{array}{c}\text { element } \\
\text { code }\end{array}$ & $\begin{array}{c}\lambda \\
(\AA)\end{array}$ & $\begin{array}{c}E W \\
(\mathrm{~m} \AA)\end{array}$ & $\log g f$ & $\begin{array}{c}E_{\text {low }} \\
(\mathrm{eV})\end{array}$ & $\log N$ \\
\hline 26.01 & 4620.52 & 85 & -3.57 & 2.828 & 7.827 \\
26.01 & 5197.58 & 114 & -2.48 & 3.230 & 7.693 \\
26.01 & 5234.63 & 115 & -2.42 & 3.221 & 7.635 \\
26.01 & 6369.46 & 43 & -4.37 & 2.891 & 7.707 \\
26.01 & 6432.68 & 71 & -3.83 & 2.891 & 7.728 \\
26.01 & 6456.38 & 85 & -2.32 & 3.903 & 7.594 \\
\hline
\end{tabular}

region, except for those lines lying within $0.03 \AA$ of the investigated line. The SYNTHE code of Kurucz (1995) was used for calculations of the synthetic spectra.

More descriptions of this type of codes for semiautomatic and automatic abundance analysis are found in Cowley (1995), Tsymbal \& Cowley (2000) (MERSEN code), Valenty \& Piskunov (1996) (SME), Erspamer \& North (2002), and Bruntt et al. (2002). Although these codes use different algorithms, they allow one to significantly increase the list of investigated lines and minimize manual work.

As initial abundances for the computation of synthetic spectrum, we use the values published in earlier investigations. This permits us to include moderately blended lines for abundance analysis and thus maximize the number of elements with determined abundances. In addition, a good guess of the initial abundances can minimize the computation time by reducing the number of iterations in the fitting process.

To compare the abundances of individual elements of HD 202109 with those of the Sun, we also calculate the solar abundances for the investigated lines. For this calculation, we use the Liége Solar atlas (Delbouille et al. 1974) and the Holweger \& Muller (1974) atmosphere model. The adopted values of the microturbulent and macroturbulent velocities are $1.0 \mathrm{~km} \mathrm{~s}^{-1}$ (Gopka \& Yushchenko 1995) and $1.8 \mathrm{~km} \mathrm{~s}^{-1}$, respectively. The continuum in the Liege Solar atlas is corrected in accordance with Arderberg \& Virdeforce (1979) and Rutten \& van der Zalm (1984). We use the same SYNTHE and URAN codes for synthetic spectrum construction, line identifications, and abundance determinations.

We present a partial list of the determined abundances of elements derived from the individual lines of HD 202109 in Table 2 (for iron lines) and 3 (for lines of other elements). Table 2 contains the element code (26.00 for FeI), wavelength, equivalent width $(E W)$, oscillator strength $(\log g f)$, excitation potential, and abundance (expressed on the scale of $\log N(\mathrm{H})=12$ ).

Table 3 contains the element code, wavelength, oscillator strength, excitation potential, abundances for $\zeta$ Cyg and for the Sun and the difference between these abundances $(\Delta)$. For lines without counterparts in the solar spectrum Grevesse \& Sauval (1998) solar system abundances were used. The full table in electronic form can be obtained at the Web sites: "users.odessa.net/ yua" and "yushchenko.netfirms.com"
Table 3. Abundances of chemical elements calculated from individual lines in the spectrum of $\zeta$ Cyg (HD 202109) and in the solar spectrum (example, full table is available in electronic form).

\begin{tabular}{|c|c|c|c|c|c|c|}
\hline \multirow{2}{*}{$\begin{array}{c}\text { element } \\
\text { code }\end{array}$} & \multirow{2}{*}{$\begin{array}{c}\lambda \\
(\AA)\end{array}$} & \multirow[t]{2}{*}{$\log g f$} & \multirow{2}{*}{$\begin{array}{l}E_{\text {low }} \\
(\mathrm{eV})\end{array}$} & \multicolumn{2}{|c|}{$\log N$} & \multirow[t]{2}{*}{$\Delta$} \\
\hline & & & & $\zeta$ Cyg & Sun & \\
\hline 72.01 & 4093.155 & -1.090 & .452 & 1.01 & .56 & .44 \\
\hline 76.00 & 3528.598 & -1.700 & .000 & 1.46 & 1.41 & .05 \\
\hline 76.00 & 3501.163 & -.510 & 1.841 & 1.96 & 1.41 & .55 \\
\hline 77.00 & 3515.947 & -1.580 & .881 & $<1.75$ & & \\
\hline 77.00 & 3594.388 & -1.550 & .881 & $<1.85$ & & \\
\hline 78.00 & 3638.789 & -1.340 & 1.254 & 1.8 & & \\
\hline 81.00 & 3519.210 & .141 & .966 & $<1.4$ & & \\
\hline 82.00 & 3683.462 & -.555 & .969 & $<2.1$ & & \\
\hline 82.00 & 3639.567 & -.715 & .969 & $<2.1$ & & \\
\hline
\end{tabular}

Table 4. CNO abundances in the atmosphere of HD 202109 with respect to their abundances in the solar atmosphere.

\begin{tabular}{ccccllll}
\hline \hline & $\begin{array}{c}\text { Sneden } \\
\text { et al. }\end{array}$ & Gratton & Berdyugina & $\begin{array}{l}\text { Cohen } \\
\text { et al. }\end{array}$ & $\begin{array}{l}\text { This } \\
\text { work }\end{array}$ & $n$ \\
& $(1981)$ & $(1985)$ & $(1993)$ & $(1999)$ & & \\
\hline $\mathrm{C}$ & -0.18 & -0.10 & -0.19 & & & \\
$\mathrm{~N}$ & +0.58 & +0.61 & +0.23 & & & \\
$\mathrm{O}$ & +0.04 & -0.34 & +0.02 & +0.35 & $-0.22:$ & 1 \\
\hline
\end{tabular}

In Tables 4 and 5, we present the final list of the abundances of 51 elements, which are computed by averaging the abundances derived from all lines of the individual elements. Table 4 contains CNO data obtained by Sneden et al. (1981), Gratton (1985), Berdyugina (1993), Cohen et al. (1999), and our result for oxygen.

The relative abundances of the other elements (listed in Table 5) are compared with the determinations of Zacs (1994) and Boyarchuk et al. (2001). Our results (except iron) were obtained with the spectrum synthesis method. The Zacs (1994) and Boyarchuk et al. (2001) data were calculated with the model atmospheres method. Elements with lines having no counterparts in the solar spectrum are marked by the asterisk. For these elements abundances are given with respect to the solar values (Grevesse \& Sauval 1998) of the corresponding elements. Also listed are the numbers of lines, $(n)$, from which the mean abundances of the individual elements are computed. The uncertainty of each element's abundance is estimated by computing the standard deviation of the abundances derived from the individual lines of the element. The uncertainties are, therefore, presented only for elements whose abundances are based on more than two lines.

We investigated the abundances of 47 elements. The abundances of $\mathrm{Li}, \mathrm{C}, \mathrm{N}$, and $\mathrm{Ba}$ are taken from the papers mentioned above. The total abundance sample consists of 51 elements. $s$-process elements show overabundances in the atmosphere of this star.

The abundances of $\mathrm{P}, \mathrm{S}, \mathrm{K}, \mathrm{Cu}, \mathrm{Zn}, \mathrm{Ge}, \mathrm{Rb}, \mathrm{Sr}, \mathrm{Nb}, \mathrm{Mo}, \mathrm{Ru}$, Rh, Pd, In, Sm, Gd, Tb, Dy, Er, Tm, Hf, Os, Ir, Pt, Tl, and Pb have been determined for the first time in this paper. 
Table 5. The abundances of chemical elements (except CNO) in the atmosphere of HD 202109 with respect to the abundances in the solar atmosphere.

\begin{tabular}{|c|c|c|c|}
\hline \multirow[b]{2}{*}{ element } & \multicolumn{3}{|c|}{$\left[N / N_{\mathrm{H}}\right](n)$} \\
\hline & $\begin{array}{c}\text { Zacs } \\
\text { (1994) }\end{array}$ & $\begin{array}{c}\text { Boyarchuk } \\
\text { et al. } \\
\text { (2001) }\end{array}$ & This work \\
\hline $3 \mathrm{Li} \mathrm{I}$ & -0.14 & & \\
\hline $8 \mathrm{O} \mathrm{I}$ & & & $-0.22: \quad$ (1) \\
\hline $11 \mathrm{Na} \mathrm{I}$ & -0.35 & +0.19 & $+0.24 \pm .08$ \\
\hline $12 \mathrm{Mg} \mathrm{I}$ & -0.51 & & $+0.22 \pm .22$ \\
\hline $13 \mathrm{Al} \mathrm{I}$ & & +0.16 & $+0.12 \pm .12$ \\
\hline $14 \mathrm{Si} \mathrm{I}$ & $+0.14 \pm .13$ & +0.09 & $-0.05 \pm .13(52)$ \\
\hline Si II & & & +0.26 \\
\hline $15 \mathrm{P} \mathrm{I}$ & & & +0.10 \\
\hline $16 \mathrm{~S} \mathrm{I}$ & & & $+0.00 \pm .12$ \\
\hline $19 \mathrm{~K} \mathrm{I}$ & & & $-0.17 \quad$ (2) \\
\hline $20 \mathrm{Ca}$ I & $+0.08 \pm .19$ & -0.03 & $+0.02 \pm .09$ \\
\hline $21 \mathrm{Sc} \mathrm{I}$ & $+0.04 \pm .30$ & -0.02 & $+0.06 \pm .11$ \\
\hline $\mathrm{Sc}$ II & & & $+0.08 \pm .15(10)$ \\
\hline $22 \mathrm{Ti} \mathrm{I}$ & $-0.17 \pm .22(21)$ & -0.11 & $-0.20 \pm .17(70)$ \\
\hline Ti II & & & $-0.03 \pm .13(30)$ \\
\hline $23 \mathrm{~V} \mathrm{I}$ & $-0.13 \pm .18(16)$ & -0.04 & $-0.03 \pm .14(41)$ \\
\hline V II & & & +0.17 \\
\hline $24 \mathrm{Cr} \mathrm{I}$ & $-0.06 \pm .18(11)$ & -0.08 & $-0.17 \pm .13(65)$ \\
\hline Cr II & & & $+0.10 \pm .10(15)$ \\
\hline $25 \mathrm{Mn} \mathrm{I}$ & $-0.30 \pm .13$ & & $-0.25 \pm .17(21)$ \\
\hline $26 \mathrm{Fe} \mathrm{I}$ & $+0.12 \pm .23(51)$ & -0.03 & $+0.01 \pm .11(89)$ \\
\hline Fe II & & & $+0.06 \pm .07$ \\
\hline 27 Co I & $-0.22 \pm .12$ & -0.13 & $+0.07 \pm .11(28)$ \\
\hline $28 \mathrm{Ni} I$ & $-0.05 \pm .25(10)$ & -0.09 & $-0.09 \pm .17(74)$ \\
\hline $29 \mathrm{Cu} \mathrm{I}$ & & & $-0.01 \quad$ (1) \\
\hline $30 \mathrm{Zn} \mathrm{I}$ & & & $-0.08 \pm .09$ \\
\hline $32 \mathrm{Ge} \mathrm{I}$ & & & +0.08 \\
\hline 37 Rb I* & & & -0.07 \\
\hline $38 \mathrm{Sr} \mathrm{I}$ & & & +0.26 \\
\hline
\end{tabular}

\subsection{Uncertainties in abundances}

The abundances of elements are subject to uncertainties caused by various sources. The uncertainties in the determined abundances caused by the uncertainty of the atmosphere model can be estimated by investigating how the iron abundance varies depending on the adopted values of atmospheric parameters. We find that the variations of the iron abundance caused by the small deviations of $\Delta T_{\text {eff }}=100 \mathrm{~K}, \Delta v_{\text {micro }}=0.1 \mathrm{~km} \mathrm{~s}^{-1}$, and $\Delta \log g=0.2$ are $0.06 \mathrm{dex}, 0.10 \mathrm{dex}$, and $0.01 \mathrm{dex}$, respectively.

Of course, the abundances of different species will have different dependencies on the perturbations in the atmosphere parameters. For example, we find that the carbon abundance estimated based on the atmosphere model adopted by Cohen et al. (1999) differs from our determination by an amount of 0.23 dex. We note, however, that our adopted atmospheric parameters of HD 202109 agree well with other determinations

\begin{tabular}{|c|c|c|c|}
\hline \multirow[b]{2}{*}{ element } & \multicolumn{3}{|c|}{$\left[N / N_{\mathrm{H}}\right](n)$} \\
\hline & $\begin{array}{c}\text { Zacs } \\
(1994)\end{array}$ & $\begin{array}{c}\text { Boyarchuk } \\
\text { et al. } \\
(2001)\end{array}$ & This work \\
\hline 39 Y I & $+0.37 \pm .24$ & +0.30 & $+0.15 \pm .15$ \\
\hline Y II & & & $+0.48 \pm .16(22)$ \\
\hline $40 \mathrm{Zr} \mathrm{I}$ & $-0.08 \pm .20$ & & $+0.21 \pm .06(10)$ \\
\hline $\mathrm{Zr}$ II & & & $+0.52 \pm .18(12)$ \\
\hline $41 \mathrm{Nb}$ II* & & & +0.43 \\
\hline 42 Mo I & & & +0.20 \\
\hline $44 \mathrm{Ru} \mathrm{I}^{*}$ & & & $+0.32 \pm .08$ \\
\hline 45 Rh I* & & & $<+0.2$ \\
\hline 46 Pd I* & & & $+0.32:$ \\
\hline 49 In I & & & $+0.11:$ \\
\hline $56 \mathrm{Ba}$ II & $+0.41 \pm .13$ & +0.54 & \\
\hline $57 \mathrm{La}$ II & $+0.38 \pm .15$ & +0.45 & $+0.51 \pm .20(12)$ \\
\hline $58 \mathrm{Ce}$ II & +0.55 & +0.33 & $+0.37 \pm .18(45)$ \\
\hline 59 Pr II & $+0.32 \pm .13$ & +0.43 & $+0.19 \pm .19$ \\
\hline $60 \mathrm{Nd}$ II & & +0.23 & $+0.42 \pm .17(70)$ \\
\hline $62 \mathrm{Sm}$ II & & & $+0.34 \pm .18(15)$ \\
\hline $63 \mathrm{Eu} \mathrm{II}$ & -0.05 & +0.22 & $+0.32 \pm .12$ \\
\hline 64 Gb II & & & $+0.27 \pm .19$ \\
\hline $65 \mathrm{~Tb} \mathrm{II}^{*}$ & & & +0.1 \\
\hline 66 Dy II & & & $+0.33 \pm .18$ \\
\hline $68 \mathrm{Er}$ II & & & +0.35 \\
\hline 69 Tm II & & & $<+0.2$ \\
\hline 72 Hf II* & & & $+0.45:$ \\
\hline 76 Os I* & & & $+0.30:$ \\
\hline 77 Ir I* & & & $<+0.4$ \\
\hline 78 Pt I* & & & +0.0 \\
\hline $81 \mathrm{Tl} \mathrm{I}^{*}$ & & & $<+0.5$ \\
\hline $82 \mathrm{~Pb} \mathrm{I*}$ & & & $<+0.2$ \\
\hline
\end{tabular}

and thus we believe that the uncertainty in the determined abundances due to the adopted atmosphere model is unlikely to be large.

If we adopt one of the parameters with some uncertainty, we try to select the other parameters to minimize correlation coefficients and scattering of the results. For example, the metallicity derived in our investigation is $+0.01 \mathrm{dex}$, and if we adopt the parameters of Boyarchuk et al. (2001) it will be changed by 0.04 dex only.

\section{Observed abundance pattern}

\subsection{CNO elements}

From Table 4, one finds that the abundances of these elements show considerable differences from one determination to another. In our analysis, we determine the abundance of 
oxygen only. We investigate the cause of the differences, focusing mainly on the difference between the oxygen abundances determined by us and by Cohen et al. (1999), for which the difference is greatest. We find several possible causes of differences.

First, the analyses are based on different oxygen lines. Our oxygen abundance is derived from line $\lambda 6300.304$. On the other hand, the oxygen abundance determined by Cohen et al. (1999) was based on triplet $\lambda \lambda 7771-7775$. Since this triplet is located in a gap in our spectra, we could not use it for the abundance analysis.

Second, the lines used for both analyses are affected by blending. The $\lambda 6300.304$ line used for our analysis is contaminated by the nickel line. Among the three lines used by Cohen et al., only the $\lambda 7771.944$ line is free from blending and the other two lines are contaminated by $\mathrm{CN}$ lines. The difference in oxygen abundances, calculated with the Cohen et al. (1999) value of the equivalent width of $\lambda 7771.944$ and calculated using spectrum synthesis for $\lambda 6300.304$ line is only 0.09 dex.

Third, the difference between the adopted atmosphere models also has some influence on the oxygen abundances. We calculated the oxygen abundance from $\lambda 6300.304$ line based on the atmosphere model adopted by Cohen et al. (1999). This abundance is higher than our original oxygen abundance by an amount of 0.23 dex.

$\mathrm{CNO}$ abundances are important for the abundance determinations of other elements. We calculated several synthetic spectra for the whole observed region with our atmosphere model parameters and different $\mathrm{CNO}$ abundances. We chose to use the values of Gratton (1985). Cratton (1985) abundances fit the observed spectra best among the determinations listed in Table 4 .

\subsection{Na to $K$}

Our results for these elements are close to the Boyarchuk et al. (2001) data for common elements. On the other hand, our values show some differences from the data of Andrievsky et al. (2002), which were released just after we completed the major part of our abundance determinations. For example, the NLTE value of the sodium abundance determined by Andrievsky et al. (2000) is +0.35 dex, compared to our value of +0.24 dex. We suspect that the differences may be caused by their adoption of an NLTE atmosphere model.

Our $\mathrm{Mg}$ abundance is in a good agreement with that of Cohen et al. (1999). Although the abundances of Si determined by Boyarchuk et al. (2001) and Cohen et al. (1999) are slightly higher than our value, all these values including ours are consistent with the solar abundance within the errors. Our results for $\mathrm{P}, \mathrm{S}$, and $\mathrm{K}$ indicate that the abundances of these elements are close to the solar values.

\subsection{Ca to Ni}

These elements produce the majority of lines in the spectra of normal stars. We find that our iron abundance, $+0.01 \mathrm{dex}$, agrees well with most previous determinations, ranging from -0.17 to +0.10 dex (Cayrel de Strobel et al. 1977). Only
Andrievsky et al. (2002) result is slightly higher: +0.15 dex. We suspect that the higher value of Andrievsky et al. (2002) is caused by the use of narrow-wavelength-range spectra in their abundance analysis. Because of this their $[\mathrm{Na} / \mathrm{Fe}]$ for HD 202109 can be higher.

We also find that the abundances derived from neutral and ionized lines result in similar values for $\mathrm{Fe}$ and $\mathrm{Sc}$, while the values of $\mathrm{Ti}, \mathrm{V}$, and $\mathrm{Cr}$ show differences of $\sim 0.2 \mathrm{dex}$.

The abundance of Mn, which is estimated by using $h f s$ data from the Kurucz (1995) data base, indicates that this element is slightly underabundant.

For elements in this group, we find that the determined abundances show a large fluctuation compared to the solar values. These fluctuations may be an intrinsic characteristic of the abundance patterns of HD 202109, or caused by inadequate sophistication of the atmospheric model.

\section{4. r-, s-process elements}

For $\mathrm{Y}$ and $\mathrm{Zr}$, we find inconsistencies between the abundances derived from the lines of neutral and ionized species. The mean result for two ions of $\mathrm{Y}$ is in perfect agreement with Boyarchuk et al. (2001) and Zacs (1994).

For line identifications of lanthanides, we use both the VALD line list (Piskunov et al. 1995) and the DREAM (Biemont et al. 2002) data base. We expected that by using the DREAM data base we could identify more lanthanide lines based on the previous experience in the spectral analysis of Przybylski's star (Yushchenko et al. 2002). For HD 202109, however, we could identify only a few more lines. We find that our results are consistent with the data of Boyarchuk et al. (2001) and Zacs (1994) for common elements.

From our investigation of the spectrum of HD 202109, we are able to determine abundances of $r$-, and $s$-process elements whose abundances were not previously known. These elements include $\mathrm{Cu}, \mathrm{Zn}, \mathrm{Ge}, \mathrm{Rb}, \mathrm{Nb}, \mathrm{Mo}, \mathrm{Ru}, \mathrm{Rh}, \mathrm{Pd}, \mathrm{In}, \mathrm{Tb}, \mathrm{Er}, \mathrm{Tm}$, $\mathrm{Hf}, \mathrm{Os}, \mathrm{Ir}, \mathrm{Pt}, \mathrm{Tl}$, and Pb. For most of these elements, we calculated the abundances based on one or two lines and for some we could only set upper limits. However, even this information is useful in constraining the parameters of the wind accretion model, which is described in the next section.

\section{Predicted heavy element abundances}

As presented and shown in Table 5 and Fig. 5, respectively, neutron-capture (n-capture) process elements (hereafter heavy elements) of HD 202109 are overabundant. Two peaks at $Z \sim$ 39-40 and $Z \sim 56-57$ are obviously due to the neutron magic numbers of 50 and 82 nuclei in the path of $s$-process nucleosynthesis occurring in the interiors of AGB stars.

What causes the heavy-element overabundances in barium stars? It is generally believed that the overabundances were caused by binary accretion, where Ba stars accreted the ejected material from their companions, the former AGB stars and the current white dwarfs, which synthesized these heavy elements by themselves and ejected the elements into the interstellar medium through the stellar wind (Liang et al. 2000 and references therein). 


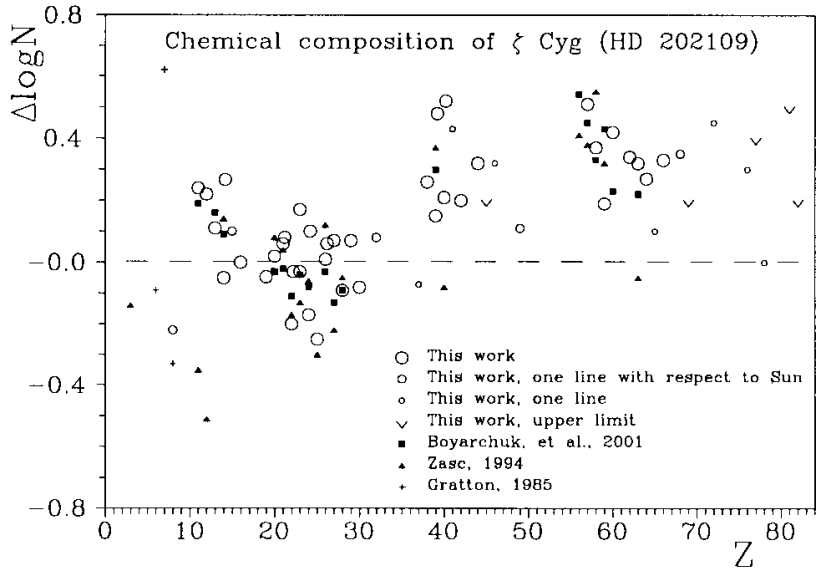

Fig. 5. The abundances of chemical elements and ions in the atmosphere of HD 202109 with respect to their abundances in the solar atmosphere. Our data and data of Boyarchuk et al. (2001), Zacs (1994), and Gratton (1985) are shown for comparison.

Liang et al. (2000) calculated the $s$-process nucleosynthesis of AGB star with mass $3 M_{\odot}$ and solar metallicity. At the same time, they set up an angular momentum conservation model of wind accretion in binary systems. Combining the AGB star nucleosynthesis and the wind accretion model, the theoretical heavy-element abundances of barium stars were calculated, and the observed abundances of some sample barium stars were explained successfully.

In this section, we check whether the binary accretion model can also explain the heavy element overabundance of HD 202109 by comparing the abundance pattern determined from our spectral analysis with the theoretical one calculated based on the model of Liang et al. (2000).

Following Liang et al. (2000), we compute the theoretical abundances of heavy elements in two steps. In the first step, we calculate the overabundances of the intrinsic AGB star at each ejection by adopting the theory of $s$-process nucleosynthesis and the latest TP-AGB model (Straniero et al. 1995; Straniero et al. 1997; Gallino et al. 1998; Busso et al. 1999). In the second step, the overabundances of heavy elements in the atmosphere of barium star are calculated by accreting the ejected matter predicted from the model of wind accretion and mixing them on successive occasions. The more details about the scenarios of $s$-process nucleosynthesis and the orbital evolution of the binary system can be found in Liang et al. (2002) and Liu et al. (2000).

The second step needs the main-sequence masses of the stars. Thus, we estimated the mass of HD 202109 by using the stellar evolution tracks given by Girardi et al. (2000). The derived mass from the $M_{\text {bol }}-\log T_{\text {eff }}$ diagram is $\sim 3.05 M_{\odot}$, which reveals that HD 202109 is a mild barium star (Jorissen et al. 1998; Liang et al. 2003).

Therefore, the adopted wind accretion model is: $3.0 M_{\odot}$ and $2.5 M_{\odot}$ for the main-sequence masses of the intrinsic AGB star (the current white dwarf companion) and the barium star, respectively; $v_{\text {ej }}=15 \mathrm{~km} \mathrm{~s}^{-1}$ for the wind velocity; 0.15 times the Bondi-Hoyle accretion rate for the actual accretion rate (Liang et al. 2002).

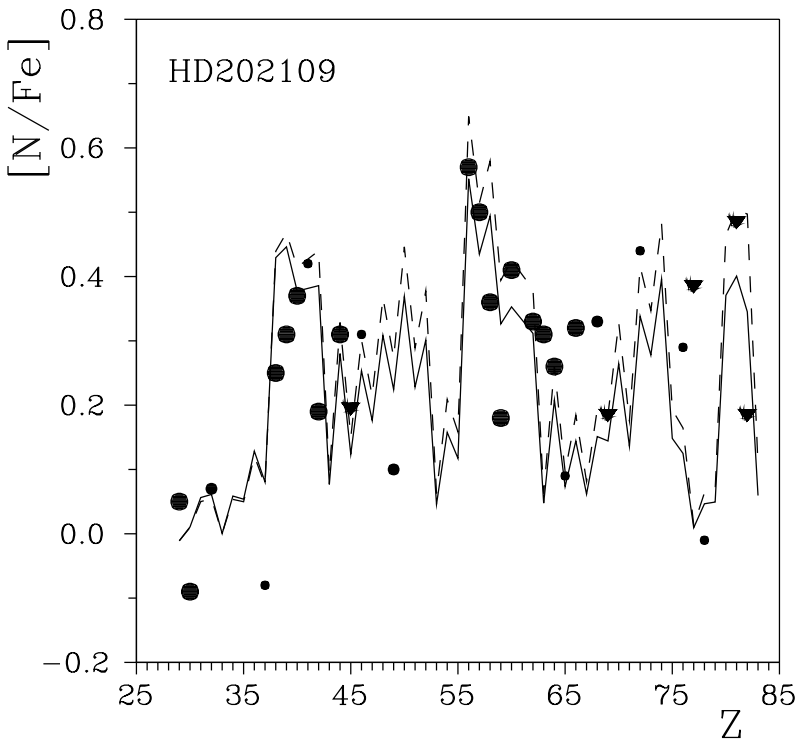

Fig. 6. The comparison of the observed composition of heavy elements in the atmosphere of HD 202109 with the predicted abundances of these elements. Solid line - calculation with $a=1.5$, dashed line $a=1.8$. Circles and triangles - observational data. For all elements except barium we plotted our results from Table 5, the Boyarchuk et al. (2001) value was plotted for barium. Big circles - more than 1 line were measured for this element, midium-sized circles - one line with respect to Sun, small circles - one line without direct comparison with the solar spectrum. Triangles - upper limit. For $\mathrm{Y}$ and $\mathrm{Zr}$ the weighted mean of the abundances from the lines of first and second spectra are plotted.

The obtained orbital eccentricity and period of the barium star system match the values $e=0.22$ and $P=6489$ days of HD 202109, respectively, which were determined observationally by Griffin \& Keenan (1992).

Figure 6 gives the theoretical abundance pattern of HD 202109 (the lines) and the pattern determined from our spectral analysis (the points). The two theoretical abundance pattern curves are the results of the cases with neutron exposures $a=1.5$ (solid curve) and 1.8 (dashed curve). " $a$ " refers to the neutron exposure occurring in its AGB star companion, and represents the times of the corresponding exposures in the ${ }^{13} \mathrm{C}$ profile suggested by Gallino et al. (1998). The more details can be found in Liang et al. (2000).

From Fig. 6, one finds that the calculated abundance pattern is consistent with the detailed pattern of the observed abundances, which means that the heavy element overabundances of HD 202109 may result from accreting the ejecta of its AGB star companion. The neutron exposure of the $s$-process nucleosynthesis occurring in the interior of the AGB companion corresponds to $a=1.5-1.8$.

\section{Conclusion}

In this paper we make a detailed analysis of abundances of $s$-process elements in the atmosphere of the mild barium star HD 202109.

The atmosphere parameters were found from the careful analysis of iron abundances, calculated from individual iron 
lines. Taking into account the variation of scattering of the mean iron abundance for atmosphere models with different parameters, we were able to develop a method to determine the effective temperature, surface gravity, microturbulent velocity and metallicity from the analysis of the iron lines.

We used differential spectrum synthesis to find the element abundances, taking the solar spectrum as a comparison one. The result is the abundances of 47 elements in the atmosphere of HD 202109. We found the abundances of several elements near the last peak of the abundances of $s$-process elements in barium stars - those near atomic number $Z=80$. The abundances of $\mathrm{Li}, \mathrm{C}, \mathrm{N}, \mathrm{Ba}$ were known from previous investigations of this star. The total abundance sample consists of 51 elements. It is the most detailed abundance pattern for barium stars now available.

We calculated the theoretical abundances of heavy elements of barium stars using AGB star nucleosynthesis and the wind accretion model in barium binary systems. The observed abundances of heavy elements of HD 202109 are consistent with the predicted abundances. It was shown that the barium star HD 202109 can be formed through a wind accretion scenario. The corresponding neutron exposure in the $s$-process nucleosynthesis in the interior of its AGB star companion is $a=1.5$ to $a=1.8$.

Barium stars with orbital period $P>1600$ days can be formed through wind accretion (Liang et al. 2002; Liang et al. 2003). Jorissen et al. (1998) suggested that the corresponding period is 1500 days. Possibly, barium stars with lower orbital period form through other scenarios: dynamically stable late case $\mathrm{C}$ mass transfer or common envelope ejection. HD 202109 ( $P=6489$ days) provides strong support for this suggestion by the consistency between the observed abundances of so many heavy elements and the corresponding predicted abundances from our wind accretion model.

Acknowledgements. We would like to thank to L. Delbouille and G. Roland for sending us the Liege Solar Atlas, to C. Han and L. Zacs for helpful discussion about the work. We use data from NASA ADS, SIMBAD, CADC, VALD, NIST, and DREAM databases and we thank the teams and administrations of these projects. The paper was (partially) supported by research funds of Chonbuk National University, Korea.

\section{References}

Alonco, A., Arribas, S., \& Martinez-Roger, C. 1999, A\&AS, 140, 261 Andrievsky, S. M., Egorova, I. A., Korotin, S. N., \& Burnage, R. 2002, A\&A, 389, 519

Aoki, W., Honda, S., Beers, T., \& Sneden, C., 2003, ApJ, 586, 506

Arderberg, A., \& Virdeforce, B. 1979, A\&AS, 36, 317

Berdyugina, S. V. 1993, Astron. Lett., 19, 378

Berdyugina, S. V., \& Savanov, I. S. 1994, Astron. Lett., 20, 755

Barstow, M. A., Bond, H. E., Burleigh, M. R., \& Holberg, J. B. 2001, MNRAS, 322, 891

Bidelman, W. P., \& Keenan, P. C. 1951, ApJ, 114, 473

Biemont, J., Palmeri, P., \& Quinet, P. 2002, Database of rare earths at Mons University // http://www . umh . ac. be/ãstro/dream. html

Boyarchuk, A., Antipova, L., Boyarchuk, M., \& Savanov, I. 2001, Astron. Rep., 45, 301
Bruntt, H., Catala, C., Garrido, R., et al. 2002, A\&A, 389, 345

Burbidge, E. M., \& Burbidge, G. R. 1957, ApJ, 126, 357

Burbidge, E. M., Burbidge, G. R., Fawler, W. A., \& Hoyle, F. 1957, Rev. Mod. Phys., 29, 547

Busso, M., Gallino, R., \& Wasserburg, G. J. 1999, ARA\&A, 37, 239

Cayrel de Strobel, G., Soubiran, C., Friel, E. D., Ralite, N., \& Francois, P. 1997, A\&AS, 124, 299

Chromey, F. R., Faber, S. M., Wood, A., \& Danziger, I. J. 1969, ApJ, 158,599

Cohen, J. C., Gratton, R. G., Behr, B. B., \& Carreta, E. 1999, ApJ, 523,739

Cowley, C. 1995, ASP Conf. Ser., 108, 170

Cowley, C. R., \& Downs, P. L. 1980, ApJ, 236, 648

Cowley, C., Ryabchikova, T., Kupka, F., et al. 2000, MNRAS, 317, 299

Delbouille, L., Roland, G., \& Neven, L. 1973, Photometric Atlas of the Solar Spectrum from $\lambda 3000$ to $\lambda 10000$ (Liège: Institut d'Astrophysique de l'Université de Liège)

de Medeiros, J. R., \& Mayor, M. 1999, A\&AS, 129, 433

Di Benedetto, G. P. 1998, A\&A, 339, 858

Erspamer, D., \& North, P. 2002, A\&A, 383, 227

Fernandez-Villacanas, J. L., Rego, M., \& Cornide, M. 1990, AJ, 99, 1961

Galazutdinov, G. A. 1992, SAO RAS Preprint No. 92

Gallino, R., Arlandini, C., Busso, M., et al. 1998, ApJ, 497, 388

Garreta, E., Cohen, J. G., Gratton, R. G., \& Behr, B. B. 2001, AJ, 122, 1469

Girardi, L., Bressan, A., Bertelli, G., \& Chiosi, C. 2000, A\&AS, 141, 371

Gopka, V. F., \& Yushchenko, A. V. 1995, Astron. Rep., 39, 662

Gray, D. F. 1976, The observations and analysis of stellar photospheres (New York: Wiley)

Gray, D. F. 1989, ApJ, 347, 1021

Gray, D. F., \& Brown, K. 2001, PASP, 113, 723

Gratton, R. G. 1985, A\&A, 148, 105

Gratton, L., Gaudenzi, S., Rossi, C., \& Gratton, R. G. 1982, MNRAS, 201,807

Grevesse, N., \& Sauval, A. J. 1998, Space Sci. Rev., 85, 161

Griffin, R. F. 1996, The Observatory, 116, 398

Griffin, R. F., \& Keenan, P. C. 1992, The Observatory, 112, 168

Gurtovenko, E. A., \& Kostik, R. I. 1989, Fraungoferov spectr i sistema solnechnikh sil oscjljatorov (Kiev. Naukova Dumka.), 198

Holweger, H., \& Muller, E. A. 1974, Sol. Phys., 39, 19

Jorissen, A., Van Eck, S., Mayor, M., \& Udry, S. 1998, A\&A, 332, 877

Keenan, P. C., \& MacNeil, R. C. 1976, An Atlas of Spectra of the Coller Stars: Types G,K,M,S, and C, Ohio State Univ.

Kovtyukh, V. V., \& Gorlova, N. I. 2000, A\&A, 358, 587

Kurucz, R. L. 1995, ASP Conf. Ser., 81, 583

Leckrone, D. S., Proffitt, C. R., Wahlgren, G. M., Johansson, S. G., \& Brage, T. 1999, AJ, 117, 1454

Liang, Y. C., Zhao, G., \& Zhang, B. 2000, A\&A, 363, 555

Liang, Y. C., Zhao, G., Chen, Y. Q., Qiu, H. M., \& Zhang, B. 2003, A\&A, 397, 257

Liu, J. H., Zhang, B., \& Liang, Y. C. 2000, A\&A, 363, 660

Malaney, R. 1987, ApJ, 321, 832

McClure, R. D., Fletcher, J. M., \& Nemec, J. M. 1980, ApJ, 238, L35

McWilliam, A. 1990, ApJS, 74, 1075

Morton, D. C. 2000, ApJS, 130, 403

Musaev, F., Galazutdinov, G., Sergeev, A., Karpov, N., \& Pod'yuachev, Y. 1999, Kinematics and Physics of Selectial Bodies, 15, 282

Pilachowski, C. A. 1977, A\&A, 54, 465 
Piskunov, N., Kupka, F., Ryabchikova, T., Weiss, W., \& Jeffery, C. Walgren, G. M. 1996. ASP Conf. Ser., 108, 240-253 1995, A\&AS, 112, 525

Pourbaix, D., \& Jorissen, A. 2000, A\&AS, 145, 161

Rutten, R. J., \& van der Zalm, E. B. J. 1984, A\&AS, 55, 143

Sneden, C., Lambert, D., \& Pilachowski, C. 1981, ApJ, 247, 1052

Sneden, C., Cowan, J. J., Ivans, I. I., et al. 2000, ApJ, 533, L139

Sneden, C., Cowan, J. J., Lawler, J. E., et al. 2003, ApJ, accepted [astro-ph/0303543]

Straniero, O., Chieffi, A., Limongi, M., et al. 1997, ApJ, 478, 332

Straniero, O., Gallino, R., Busso, M., et al. 1995, ApJ, 440, L85

Tarasova, T. N. 2002, Astron. Rep., 46, 474

Tsymbal, V., \& Cowley, C. 2000, private communication

Valenty, J. A., \& Piskunov, N. E. 1996, A\&AS, 118, 595

Wallerstein, G., Iben, I., Parker, P., et al. 1997, Rev. Mod. Phys., 69, 995

Yushchenko, A. V. 1998, Proc. of the 29th conf. of variable star research, Brno, Czech Republic, November 5-9, 201

Yushchenko, A. V., \& Gopka, V. F. 1996a, Astron. Lett., 22, 412

Yushchenko, A. V., \& Gopka, V. F. 1996b, Odessa Astron. Publ., 9, 86

Yushchenko, A. V., Gopka, V. F., Khokhlova, V. L., Musaev, F. A., \& Bikmaev, I. F. 1999, Astron. Lett., 25, 453

Yushchenko, A., Gopka, V., Kim, C., et al. 2002, J. Korean Astron. Soc., 35, 209

Zacs, L. 1994, A\&A, 283, 937 